\section{Asthma induced by epoxy resin systems}

We are all environmentalists nowadays, but an int erest in the air we breathe has long been of concern to chest physicians, particularly in the management of asthmatic patients. In addition to the common atmospheric and domestic allergens, patients may be exposed in their work to chemicals such as the isocyanates, piperazine, and formalin, which may provoke asthmatic attacks. More recently, interest has been focused on the epoxy resin systems. ${ }^{12}$

Epoxy resin systems are used in industry as adhesives, reinforced plastics, moulding resins, and surface coatings. The resins are converted to the finished product by adding curing agents or hardeners. When curing agents such as the acid anhydrides are used the process requires additional heat, while when cold curing agents such as the amine compounds are used the process generates its own heat. In either case, fumes of curing agent may be given off and may provoke asthma in a susceptible person.

This asthmatic response appears to be a true hypersensitivity, ${ }^{13}$ for there is no reaction on first exposure, but after a latent period asthma develops on exposure to low concentrations, though only in a minority of the exposed population. The bronchial reaction may be immediate, nonimmediate, or dual, and may be elicited by a few breaths of the sensitising fumes. We still do not know the precise immunological mechanisms, but Maccia et al $^{4}$ reported an instance of phthalic acid anhydride sensitivity in which the patient had a raised titre of specific IgE. Another patient did not have a raised titre, ${ }^{2}$ but the delayed part of the dual bronchoconstrictor response was inhibited by pretreatment with sodium cromoglycate.

A careful history, with particular attention to chemical processes at work, will usually alert the physician to the possibility that asthma is occupational. The typical pattern is of wheeze occurring either immediately or several hours after exposure with partial or complete recovery at weekends and during holidays. The latent period between first exposure and asthma may vary from a few days to months or even years. A known exposure to a known causative agent makes the diagnosis more likely, while objective confirmation of an occupational cause may sometimes be obtained by asking the patient to keep a work diary and record his peak expiratory flow three or four times a day. Absolute identification of the precise cause requires referral for careful bronchial provocation testing, ${ }^{5}$ when a fall in peak flow or airway conductance after inhalation of controlled amounts of the suspected agent confirms the diagnosis. The procedure is potentially hazardous-a severe attack of asthma may ensue-and should be undertaken only in hospital, with observation overnight in case a delayed reaction occurs.

Provocation testing may help in individual management by confirming a suspected cause and may occasionally show that the putative cause is innocent. Further testing may incriminate some unsuspected agent. Provocation tests are also important in identifying new causes of occupational asthma and may prove of value in clarifying the mechanisms responsible.

Acute attacks of asthma provoked by epoxy resin systems respond to the usual treatment with bronchodilators. Systemic steroids should be added if the attack is severe or protracted. Sodium cromoglycate and inhaled steroids may help to control recurrent or continuous asthma. Clearly, however, the best treatment is complete avoidance of exposure, which may require a change of occupation. Such advice should not be given lightly, and if possible it should be backed by objective evidence of an occupational factor identified by formal provocation testing. With complete exclusion of the precipitating cause the asthma may resolve completely, and the outlook is then excellent. In contrast, continuing exposure may cause increasing sensitisation with more severe and chronic asthma requiring continuous suppressive treatment, leading eventually to permanent deterioration of lung function.

\footnotetext{
${ }^{1}$ Fawcett, I W, Newman Taylor, A J, and Pepys, J, Clinical Allergy, 1977, 7,1

2 Chester, E H, et al, Clinical Allergy, 1977, 7, 15.

${ }^{3}$ Dernehl, C U, Fournal of Occupational Medicine, 1963, 5, 17.

4 Maccia, A C, et al, American Review of Respiratory Diseases, 1976, 113, 701.

${ }^{5}$ Pepys, J, and Hutchcroft, B J, American Review of Respiratory Diseases, $1975,112,829$.
}

\section{Soft contact lenses}

Recently there has been a sharp swing towards the use of soft, as opposed to hard, contact lenses. This has come about because these can be worn for longer periods, and soft lenses are now widely used in three different sorts of conditions: for low refractive error in normal eyes, in aphakia, and as a protective membrane in many corneal disorders. After eliminating any discussion of the third category, we may ask how far the results of wearing soft contact lenses for the first two compare with those of other established methods of optical correction.

Low refractive error in normal eyes may, of course, be corrected by spectacles, but many patients prefer contact lenses because of their convenience, efficiency, and cosmetic appearance. In this group soft contact lenses therefore have to be compared with hard contact lenses. To take the disadvantages: firstly, most eyes cannot tolerate them for extended periods even with the high water content Sauflon 70 and Sauflon 85 lenses. There is a limit to the degree of hypoxia that the endothelium of the cornea will tolerate. Secondly, even if a particular eye can tolerate extended wear, however, there is still a danger of bacterial or fungal keratitis. The latter may be disastrous and may be masked by the lens while it remains in wear. Thirdly, complicated routines for cleaning the lenses are necessary. Fourthly, the lenses are fragile and they tend to cost more than hard lenses. Fifthly, when a lost or damaged soft lens is replaced by another apparently identical lens both the comfort and vision may be poorer than with the first lens. Soft lenses lack the reproducibility of hard lenses. On the other hand, soft lenses do have certain definite advantages for low refractive errors in normal eyes: they are easier to get used to than hard lenses, and they are more easily managed by the intermittent wearer.

In aphakia optical correction by spectacle lenses admittedly has certain disadvantages. Nevertheless, it is wrong to say that patients are seldom happy with their post-cataract glasses, since after an initial period of learning the great majority manage very well indeed. This fact is forgotten, however, by those who advocate optical correction by intraocular acrylic lenses or extended-wear soft contact lenses. Both methods have inherent dangers, which are avoided with conventional spectacles. Intraocular acrylic lenses and soft contact lenses should therefore be compared in the knowledge that both introduce avoidable dangers. Even when introduced skilfully, 